\title{
Marco Conceptual para la Definición del Desarrollo Sustentable (a)
}

\author{
A Sustainable Development \\ Defining Framework
}

Ricardo Goñi ${ }^{1}$

Francisco Goin ${ }^{2}$

\footnotetext{
${ }^{1}$ Licenciado en Biología. Profesor de la Cátedra Medio Ambiente y Salud, Facultad de Ciencia y Tecnología, Universidad Autónoma de Entre Ríos (UADER), Argentina.

rgcaro@ciudad.com.ar

${ }^{2}$ Licenciado en Biología. Doctor en Ciencias Naturales. Investigador CONICET,

Departamento Paleontología Vertebrados, Museo de Ciencias Naturales de La Plata, Argentina. fgoin@way.com.ar
}

RESUMEN Se sintetiza una concepción del desarrollo sustentable elaborada por los autores de este trabajo desde una perspectiva multidimensional (social, económica y ambiental), fundamentalmente signada por una impronta político-social y una visión crítica del "sustentabilismo" ecologista hegemónico.

PALABRAS CLAVE Ecología Humana; Medio Social; Desarrollo Sostenible (b).

ABSTRACT We sinthetisize a conception of sustainable development previously stated by us, based on a multidimensional (social, economic and environmental) perspective, and signed by a specific political-social departure, as well as a critic vision of the hegemonic "green" sustainability concepts.

KEY WORDS Human Ecology; Social Environment; Sustainable Development. 


\section{INTRODUCCIÓN}

En 1987 la Comisión Mundial para el Medio Ambiente y el Desarrollo Humano aprobó por unanimidad un documento denominado "Nuestro Futuro Común" (2), el cual constituyó un punto de inflexión en el debate a nivel global sobre medio ambiente y desarrollo. Allí se definió por primera vez el término desarrollo sustentable, si bien su utilización se remonta a la década anterior. En un sentido general, el desarrollo sustentable es "un proceso que busca satisfacer las necesidades humanas, tanto de las generaciones actuales como futuras, sin que ello implique la destrucción de la base misma del desarrollo, es decir, los recursos naturales y los procesos ecológicos" (3). Sin embargo, pese a que ha sido monótonamente citado en infinidad de documentos (económicos, políticos, científicos, etc.) a lo largo de los últimos veinte años, el concepto de sustentabilidad es ambiguo, a juzgar por las múltiples y contradictorias acepciones con que ha sido utilizado. ¿Qué significado tiene para la ONU, para el FMI o para el ecologismo el desarrollo sustentable? ¿Qué quiere decir "satisfacer las necesidades humanas"? ¿Cuáles son los patrones a seguir para la definición de estrategias de sustentabilidad?

Son muchos los interrogantes aún no resueltos en torno al marco conceptual para definir con más precisión el desarrollo sustentable. Como punto de partida, sin embargo, una de las cuestiones básicas es saber qué es lo que se quiere sustentar: el ambiente, o la economía, o algún recurso natural, o la sociedad y su entorno, por sólo mencionar algunas alternativas. En este trabajo se resume una concepción del desarrollo sustentable elaborada por los autores de este trabajo, con particulares referencias a la Argentina $(4,5)$. Lejos de ser políticamente "aséptica" -como ocurre con gran parte de los informes académicos- dicha concepción está fuertemente signada por una visión con una fuerte impronta social y fundamentalmente crítica a los modelos capitalistas hegemónicos.

\section{EL SIGNIFICADO PROFUNDO DE LA SUSTENTABILIDAD}

Bajo la aparente "neutralidad ideológica" de los documentos técnicos sobre el desarroIlo sustentable elaborados por las Naciones Unidas y otras agencias internacionales, se suelen resaltar cuestiones propias de los intereses de las naciones y élites firmantes de estos documentos, a la vez que se ocultan las tensiones y contradicciones que ellos generan sobre los países del Tercer Mundo. Esta es una cuestión de fundamental importancia en la comprensión del debate sobre la sustentabilidad. Por ejemplo, en un documento de las Naciones Unidas, presentado en 2001, en la novena sesión (16 al 27 de Abril de 2001) de la Comisión de Desarrollo Sustentable del Departamento de Asuntos Sociales y Económicos, se llegó a afirmar: "el libre comercio y el desarrollo sustentable se apoyan mutuamente" (6). ¿De dónde surgió esto? Tal vez de los canales secundarios del delta del Rin, donde se han "restaurado" los ecosistemas ribereños, probablemente los más subsidiados del mundo, en donde abundan espíritus "verdes" emocionados observando las "salvajes" tropillas de caballos polacos introducidos en la zona. O quizá de las chacras españolas donde se alimenta a los cerdos con almendras y avellanas para la producción del jamón de Jabudo. Pero no de las selvas taladas del Amazonas. No de los campos de monocultivo de soja en la Argentina.

Por ello, la discusión sobre el marco conceptual del desarrollo sustentable, lejos de tratarse de una cuestión formal, es fundamental para avanzar en un aspecto clave, frecuentemente soslayado: en su concepción más amplia, la sustentabilidad es un término aplicable a la sociedad en su conjunto, no a sectores específicos (e.g., "el ambiente", "la política", "la economía"). Desde esta perspectiva, no existe desarrollo sustentable real si no se identifica al cuerpo social como beneficiario final del mismo. Esto tiene consecuencias no previstas en la mayor parte de los ensayos actuales sobre el tema, ya que propone una nueva serie de prioridades en la implementación de los planes de desarrollo: la sustentabilidad aquí concebida implica, en primer lugar, la resolución inmediata de la miseria a 
nivel mundial y la atención prioritaria al desarroIlo de condiciones de vida dignas para la totalidad de la población.

En otras palabras, el debate sobre otro tipo de desarrollo -ya que de ello se trata la discusión sobre la sustentabilidad- no puede ni debe ignorar que en los países "en vías de desarrollo", como se suele denominar a los países pobres, las condiciones actuales (socioeconómicas y ambientales) se pagan con desnutrición, enfermedades, analfabetismo y más subdesarrollo. Lo contrario significa hacerse eco de la perversión semántica de los documentos sobre el desarroIlo sustentable elaborados por la ONU o las organizaciones ecologistas, cosa que no puede ocurrir entre los habitantes del Tercer Mundo, donde se convive diariamente con los resultados prácticos de políticas profundamente sesgadas en lo social.

Desde esa perspectiva, las medidas más estrictamente "ecologistas" (frecuentes usuarios del término "sustentabilidad") no constituyen un parámetro de éxito en el camino al desarrollo sustentable, en la medida en que las condiciones de vida de la población no superen umbrales mínimos de calidad de vida. El continente africano, por ejemplo, es receptor de enormes aportes económicos para la conservación de elefantes, leones, gorilas de montaña o los ecosistemas de sabana. Nada de esto queda reflejado en la población africana, la de peor calidad de vida del mundo en términos tanto cuantitativos (e.g., PBI, aporte nutricional per capita) como cualitativos (e.g., calidad de sus instituciones). Aquí quedan reflejadas con todo dramatismo las limitaciones (las perversiones) del pensamiento ambientalista o ecologista en boga: "salvemos los elefantes", "salvemos los gorilas", nunca "salvemos a los africanos". En la práctica, para estas organizaciones los africanos son más bien un recurso para la protección de elefantes o gorilas, nunca un objetivo en sí mismo. La extinción de poblaciones africanas enteras por hambre no parece afectar la conciencia de los ambientalistas, quienes remiten la ayuda a "organizaciones humanitarias". Para esta concepción, la desnutrición africana, el colapso de sus economías, es un ámbito adecuado para la acción de la Cruz Roja, Oxfam o Médicos sin Fronteras, nunca tierra fértil para el pensamiento ecologista propiamente dicho. Los fracasos del desarrollo sustentable en todo el mundo, pero especialmente en el Tercer Mundo, obedecen en parte a una incomprensión elemental de ese concepto.

\section{PISOS O ETAPAS SUCESIVAS DEL DESARROLLO}

¿Qué valor tiene lo anteriormente señalado? En primer lugar, la aplicación de un concepto de sustentabilidad como el aquí discutido propone una planificación del desarrollo con una característica básica: su proyección en el largo plazo. Quienes piensen el desarrollo sustentable deben pensar en las generaciones por venir, no en el año que viene. Este rasgo elemental tiene varios corolarios políticos de importancia:

a. la necesidad de pensar el futuro sin rigideces ni preconceptos que puedan volverse obsoletos por las circunstancias de coyuntura;

b. la obligación de anticiparse a los cambios y tendencias de mayor envergadura a partir de los datos del presente $y$

c. en el plano de la ejecución de políticas de desarrollo, la necesidad de establecer pactos generacionales que permitan la continuidad en el tiempo de las políticas de largo plazo.

En segundo lugar, su valor radica en que otorga prioridades a la planificación de acuerdo con metas prefijadas de progreso, progreso medido no en unidades de infraestructura, no en hectáreas de ecosistemas restaurados, no en kilómetros de rutas asfaltadas, sino en unidades de progreso social. La planificación de estas metas de progreso incluye el establecimiento de objetivos que, en el corto plazo, podrían incluso resultar poco sustentables a los ambientalistas contemporáneos. La decisión de talar determinados sectores selváticos para la implementación de sistemas agrícolas que hagan frente a una población que todavía no ha alcanzado su estabilidad demográfica, por dar un ejemplo muy gráfico, sería una de esas medidas. Si alimentarse constituye una medida de progreso, entonces deberán diseñarse objetivos de corto plazo que permitan alcanzar dicha meta. Esto es, el paradigma de sustentabilidad 
aquí definido permite fijar pisos sucesivos o etapas de desarrollo que son cambiantes en la medida en que progresa la sociedad misma y su entorno (natural y artificial).

La identificación de pisos sucesivos de desarrollo impone prioridades a la planificación de acuerdo con metas prefijadas de progreso social. Por lo tanto, los principales lineamientos de una política de desarrollo sustentable deberían enfatizar al principio aquellos aspectos que permitan mitigar primero, y revertir después, las causas y consecuencias de la presente desigualdad social. Especial énfasis merecen entonces aqueIlas medidas de planificación cuyo objetivo central sea el mejoramiento sustancial de las condiciones de vida de los sectores marginalizados de la economía formal, los vastos sectores que integran el universo de los "nuevos pobres" $y$, finalmente, los grupos etarios más vulnerables de la sociedad: niños y ancianos. Esto no implica abandonar criterios de sustentabilidad en los aspectos más típicamente ambientales a favor de necesidades sociales más urgentes (el deterioro ambiental es sufrido particularmente por los sectores sociales más marginales) sino proponer un eje de prioridades políticas a partir del cual se articulen otros aspectos o metas de planificación. En tiempos "normales" esto no debería ser necesariamente así. Hoy sí. Si lo que se quiere sustentar es la sociedad y su entorno, la muerte por inanición, o por enfermedades evitables o por violencia social constituyen los factores principales de insustentabilidad. Para graficarlo más adecuadamente: hoy, la idea-fuerza más importante a la que tiene que apuntar la definición de la sustentabilidad debe ser (más que "Argentina: territorio libre de contaminación ambiental") "Argentina: un País donde la gente no se muere de hambre".

En función de lo enunciado anteriormente, los autores de este trabajo diseñaron un modelo de indicadores del desarrollo sustentable para la Argentina a fin de monitorear tal proceso a lo largo del tiempo (7). A diferencia de los modelos generados por organismos internacionales, como la ONU, y por la mayor parte de los países que los han adoptado, se propuso un conjunto de indicadores que permita acompañar la evaluación de un proceso de desarrollo acotado a dichos pisos o etapas sucesivas. El modelo de indicadores propone varios objetivos: por un lado, dar prioridad a criterios de éxito que tomen como "blanco" a la mitad de la población argentina que está bajo la línea de pobreza, esto es, a la resultante social de la Argentina de la década de 1990. En segundo lugar, promover y alentar un tipo de desarrollo por etapas, con un fuerte componente de planificación estatal y al crecimiento "para adentro" (i.e., el mercado interno) tan fuerte o más que el crecimiento "para afuera" (i.e., exportaciones). Finalmente, asumir compromisos sostenidos de sustentabilidad para el crecimiento futuro.

En términos de sustentabilidad estrictamente ambiental, el modelo de indicadores antes mencionado va de menor a mayor, esto es, privilegia un estilo de desarrollo menos sustentable (en lo ambiental) en la fase inicial y más sustentable en la última de estas etapas. El modelo de indicadores propone un devenir de desarrollo que dé cuenta de tres momentos definidos para el desarrollo nacional:

a. la actual emergencia social, en donde las acciones a emprender por parte del aparato del Estado deberían apuntar a mitigar primero, y corregir y eliminar después, las causas y consecuencias de la debacle social heredada de las décadas anteriores y especialmente de la década del '90;

b. una etapa de consolidación de un tipo de desarrollo claramente planificado y con importante participación estatal, y

c. una etapa de "sustentabilidad" efectiva del desarrollo, la que supone que los problemas básicos de subsistencia han sido estructuralmente superados y la infraestructura productiva del país está en marcha.

Por ende, los temas prioritarios deben ser definidos en torno a la promoción de innovaciones tecnológicas relacionadas con la conservación del ambiente y la "despetrolización" de la producción y su reemplazo por fuentes energéticas alternativas.

Vale la pena reiterar que la primera etapa no invalida a la segunda ni a la tercera, de igual modo que ocurre con la segunda en relación a la tercera. En realidad, la atención de la emergencia social debe ir acompañada por el diseño de una estrategia de crecimiento y desarrollo. 
Vale decir, que estas dos etapas se complementan y solapan parcialmente, por ejemplo en el caso de la inversión en infraestructura, adecuada para la primera por su carácter de mano de obra intensiva pero que servirá de soporte logístico básico en la segunda etapa. Por último, al igual que las anteriores, la etapa de sustentabilidad complementa a la de consolidación. En síntesis, cada etapa define prioridades pero no implica el abandono de las restantes; a su vez, cada una de ellas no son compartimentos estancos, sino que se complementan entre sí y están sujetas a cambios permanentes.

\section{¿CATÁSTROFE O NUEVA SOCIEDAD?}

En 1977 un grupo de intelectuales y científicos de la Fundación Bariloche (Argentina) publicó un informe titulado ¿Catástrofe o nueva sociedad? Modelo Mundial Latinoamericano (7), también conocido como Informe Bariloche. Su finalidad fue demostrar que el futuro de la humanidad no dependía de barreras físicas insuperables, tal como se afirmaba en Los Límites del Crecimiento (8) (un informe elaborado por científicos del Instituto Tecnológico de Massachusetts, MIT, y auspiciado por el Club de Roma), sino de transformaciones políticas y sociales. El Informe Bariloche fue una crítica técnica, filosófica y ética al modelo del Club de Roma (considerado el primer modelo mundial). Mientras que éste, desde una perspectiva neomalthusiana, pronosticaba el colapso planetario en virtud del carácter limitado de los recursos, el crecimiento demográfico y la contaminación industrial, el informe Bariloche centró su análisis en las asimetrías entre los países ricos y pobres, en la desigual distribución del poder y los recursos, tanto a nivel internacional como dentro de cada país, y en la necesidad de lograr un mundo donde la población en su conjunto alcance mejores condiciones de vida.

A diferencia del modelo del MIT (basado en la proyección lineal de variables), el Informe Bariloche era explícitamente normativo:

"Cualquier pronóstico a largo plazo sobre el desarrollo de la humanidad se funda en una visión del mundo basada en un sistema de valores y en una ideología concreta. Suponer que la estructura del mundo actual y el sistema de valores que la sustenta pueden ser proyectados sin cambio hacia el futuro, no es una visión 'objetiva' de la realidad, como a veces se sostiene, sino que implica también una toma de posición ideológica. Por eso, la diferencia que suele establecerse entre modelos proyectivos y normativos a largo plazo es esencialmente falaz (...) El modelo que aquí se presenta es explícitamente normativo: no se ocupa de predecir qué ocurrirá si continúan las tendencias actuales de la humanidad, sino de señalar una manera de alcanzar la meta final de un mundo liberado del atraso y la miseria" (7 p.11).

Los autores del informe no ocultaron su identidad ideológica tras las máscaras de la "objetividad" y "neutralidad" de la ciencia. En tal sentido señalaban:

"[El modelo] no pretende ser 'objetivo' en el sentido valorativamente neutro con el que frecuentemente se usa esa palabra; representa la concepción del mundo que comparten sus autores y con la cual se hallan profundamente comprometidos. Es objetivo, sí, en el sentido que parte de una visión realista y descarnada de los problemas del mundo actual, y trata de encontrar soluciones basadas en la capacidad de cambio y creación tantas veces demostrada en el pasado por la sociedad humana" (7 p.11).

El estudio consistió, por un lado, en la definición de un modelo conceptual como propuesta de una nueva sociedad (sociedad ideal) y, por el otro, en el desarrollo de un modelo matemático como instrumento para contrastar su viabilidad práctica. Las premisas generales sobre las que se basó la sociedad propuesta fueron las siguientes:

a. El modelo del MIT predice catástrofes en un futuro cercano. En realidad, la catástrofe es ya una realidad cotidiana para gran parte de la humanidad.

b. Los países del Tercer Mundo no pueden progresar copiando las pautas seguidas en el pasado por los países actualmente desarrollados.

c. Las políticas de preservación de los ecosistemas o de reducción del consumo serán difíciles de ejecutar efectivamente hasta que la totalidad de la población logre un nivel de vida aceptable.

d. Los sectores privilegiados de la humanidad, esencialmente los países desarrollados, deben 
disminuir su tasa de crecimiento económico para aliviar su presión sobre los recursos naturales y para contrarrestar los efectos alienantes del consumo excesivo.

Finalmente, las características más generales de la sociedad propuesta eran las siguientes:

a. igualitaria, tanto social como internacionalmente;

b. no consumista, y donde la producción esté determinada por las necesidades sociales y no por la ganancia, y

c. con un cambio en la significación del concepto actual sobre la propiedad.

En relación a la última característica se señala:

"El concepto corriente de propiedad debe ser reemplazado por el más universal de uso de los bienes de producción y de la tierra. No existiría propiedad de estos bienes sino gestión de los mismos, decidida y organizada por los mismos procesos de discusión mediante los cuales se regula el resto de las actividades sociales. La gestión correspondería a las organizaciones de producción, a entes comunitarios ad hoc, a las comunas o al Estado, según fuera la naturaleza y el nivel de la actividad considerada" (7 p. 33).

Los puntos más sobresalientes de las conclusiones del informe son los siguientes:

1. "El modelo muestra que el crecimiento de la población puede controlarse, hasta alcanzar el estado de equilibrio, mediante la elevación general de las condiciones de vida, especialmente las relacionadas con las necesidades básicas. Ahora bien, este equilibrio se puede alcanzar en el plano mundial mucho antes de que se llegue a saturar la capacidad de producir alimentos, aun suponiendo que se siga basando en las tecnologías actualmente en uso."

2. "Se comprueba, además, que los obstáculos que actualmente se oponen a un desarrollo armónico de la humanidad no son físicos o económicos, en el sentido estricto, sino esencialmente sociopolíticos."

3. "Las tasas de crecimiento de la economía requeridas para conseguir los objetivos deseados -y que pueden obtenerse fácilmente sin imponer sacrificios sociales intolerables- contrastan con las que serían necesarias para satisfacer, aproximadamente en el mismo plazo, las necesidades básicas manteniendo la estructura actual del ingreso y, por ende, la misma organización económico-social. Estas tasas de crecimiento económico que deberían oscilar, para los países subdesarrollados entre el 10 y casi el 12\%, son inalcanzables en la realidad. El plantear este tipo de 'solución', por lo tanto, sólo tiene como objetivo preservar el actual statu quo y disimular las verdaderas causas de la crisis que afecta al mundo contemporáneo." (...)

4. "Por último, el modelo demuestra, dentro de las limitaciones que necesariamente tiene este tipo de trabajo, que el destino humano no depende, en última instancia, de barreras físicas insuperables, sino de factores sociales y políticos que a los hombres compete modificar. Nada fácil es la solución, porque cambiar la organización y los valores de la sociedad, como lo prueba la historia, es mucho más difícil que vencer las limitaciones físicas. Intentarlo, sin embargo, es el único camino abierto hacia una humanidad mejor" (7 p.123-125).

El informe concluye con una reflexión:

"Se podría decir que esta propuesta es utópica, y que sería más realista postular soluciones que implicaran una modificación menos radical de las estructuras sociopolíticas del mundo. A quienes sostienen esa posición, cabe recordar lo que escribiera John Stuart Mill hace ya un siglo: Contra un gran mal, un pequeño remedio no produce un pequeño resultado, simplemente no produce ningún resultado" (7 p.125).

\section{COROLARIO}

El Informe Bariloche fue revolucionario. Sin embargo (o quizá por ello), nunca contó con el apoyo de la comunidad académica nacional ni internacional, quien mayoritariamente se inclinó de manera explícita hacia su contracara ideológica: la visión neomalthusiana del Club de Roma. Casi treinta años después, en 2004, se 
publicó -no con mayor éxito, por lo que puede constatarse- la segunda edición del Informe Bariloche. Allí, algunos de los autores del Informe original reflexionan sobre el mismo. Uno de ellos, Enrique Oteiza, señala: "El título del libro ¿Catástrofe o Nueva Sociedad? refleja adecuadamente que, de acuerdo a los resultados obtenidos a través del Modelo Mundial Latinoamericano, la única salida posible a las tendencias del proyecto hegemónico vigente consistía en una estrategia alternativa. Esta conclusión es aún válida en el momento actual, dadas las condiciones socioeconómicas y del medio ambiente existentes en las diferentes regiones del planeta" (9), conclusión que es compartida por los autores de este trabajo, para quienes el Informe Bariloche constituyó un aporte paradigmático, desde el punto de vista teórico, en la elaboración del marco conceptual del desarrollo sustentable.

A juicio del ecólogo argentino Gilberto Gallopín, si se tiene en cuenta que el mismo camino considerado insustentable en 1992 por unos 100 mandatarios en la Cumbre de la Tierra de Río de Janeiro, es en la actualidad proclamado como un ascenso triunfal por el Banco Mundial y otras organizaciones financieras internacionales, entonces, la situación actual del mundo es "... un tanto surrealista sino esquizofrénica" (9). En ese marco, "liberar al mundo del atraso y la miseria, y revertir la situación de explotación del hombre y el medio ambiente mediante un cambio radical en la organización social e internacional del mundo", tal como lo propone el Modelo Mundial Latinoamericano de la Fundación Bariloche, parecería una visión "poco objetiva" y sin fundamentos científicos de la realidad. Sin embargo, ¿es legítimo pensar que el futuro de la humanidad está signado por la proyección del sistema de valores y la organización social vigentes? Aunque las tendencias actuales no son muy alentadoras, cabe recordar, finalmente, un párrafo de La Civilización puesta a prueba de Arnold Toynbee:

"Observada desde este mirador histórico que es el año 1947, parece una locura la alucinación fin de siécle que padeció la clase media inglesa. Ella, sin embargo, fue padecida también por la clase media de otras naciones de Occidente. En los Estados Unidos, por ejemplo, en el Norte, la clase media creía que la historia había finalizado con la conquista del Oeste y el triunfo federal de la guerra de Secesión. Lo mismo ocurrió en Alemania -o por lo menos en Prusia- donde para esa clase se había obtenido la misma eterna consumación mediante la derrota de Francia y la fundación del Segundo Reich en 1891. Para estas tres hornadas de personas pertenecientes hace cincuenta años a la clase media occidental, la obra creadora del Señor se había completado, '...y era buena'. Pero aunque en 1897 la clase media inglesa, americana y alemana fuera, tomada en su conjunto, el amo político y económico del mundo, su número apenas llegaba a una reducida fracción de la generación entonces vigente de la humanidad; y había quienes en el extranjero, aunque impotentes y sin hacerse oír, veían las cosas de distinto modo" (10).

\section{NOTAS FINALES}

a. Parte de los contenidos de este artículo han sido publicados en el libro Desarrollo Sustentable en Tiempos Interesantes. Contextos e Indicadores para la Argentina (1).

b. Nota de los editores: el Cuerpo Editorial ha decidido respetar el descriptor Desarrollo Sostenible en función de cumplir con el índice de Descriptores en Ciencias de la Salud creado por BIREME. Sin embargo los autores resaltan la existencia de una diferencia conceptual entre Desarrollo Sustentable y Desarrollo Sostenible, señalando que "mientras el primer término se refiere al desarrollo desde una perspectiva integral; el segundo, en cambio, alude más a lo estrictamente ambiental", por lo tanto, el término más apropiado sería, en este caso, Desarrollo Sustentable. 


\section{REFERENCIAS BIBLIOGRÁFICAS}

1. Goñi R, Goin F. El Desarrollo Sustentable en Tiempos Interesantes. Contextos e Indicadores para la Argentina. Buenos Aires: Scalabrini Ortiz; 2006.

2. World Comision on Environment and Development. Our Common Future. Oxford, New York: Oxford University Press; 1987.

3. Winograd M. Marco Conceptual para el Desarrollo y Uso de Indicadores Ambientales y de Sustentabilidad para la Toma de Decisiones en Latinoamérica y el Caribe. Proyecto CIATPNUMA. Cali: CIAT; 1995. [Documento de Discusión CIAT].

4. Goñi R, Goin F, Galliari C. Indicadores de Sustentabilidad y Procesos de Desarrollo. El caso de La Plata, Berisso y Ensenada. Buenos Aires: Fundación Encuentro Bonaerense; 2001. (Serie Desarrollo y Medio Ambiente).

5. Goñi R, Goin F. Los indicadores del desarrollo sustentable. Definición de un modelo para la Argentina. Buenos Aires: Consejo Federal de Inversiones; 2004. [Informe Inédito].
6. ONU. Department of Economic and Social Affairs. Indicators of Sustainable Development: Framework and Methodologies. Procedente de la Commission on Sustainable Development, Ninth Session; 16-27 April 2001. [Background paper No3]. New York: Division of Sustainable Development, UN; 2001.

7. Herrera AO, Scolnik HD, Chichilnisky G, Gallopín GC, Hardoy JE, Mosovich D, et al. ¿Catástrofe o Nueva Sociedad? Modelo Mundial Latinoamericano. Ottawa: IDRC; 1977.

8. Meadows DH, Meadows DL, Randers J. Los Límites del Crecimiento. Informe del Club de Roma sobre el Predicamento de la Humanidad. México: Fondo de Cultura Económica; 1972.

9. Herrera AO, Scolnik HD, Chichilnisky G, Gallopín GC, Hardoy JE, Mosovich D, Oteiza E, de Romero Brest G, Suarez CE, Talavera L. ¿Catástrofe o Nueva Sociedad? Modelo Mundial Latinoamericano. Treinta Años Después. 2da ed. Ottawa: IDRC-CRDI; 2004.

10. Toynbee AJ. La civilización puesta a prueba. Buenos Aires: Emecé Editores; 1949.

\section{FORMA DE CITAR}

Goñi R, Goin F. Marco Conceptual para la Definición del Desarrollo Sustentable. Salud Colectiva. 2006;2(2):191-198.

Recibido el 6 de noviembre de 2005

Versión final presentada el 21 de abril de 2006

Aprobado el 5 de mayo de 2006 